\title{
Evaluating the Validity and Reliability of Harvard Step Test to Predict VO2max in Terms of the Step Height According to the Knee Joint Angle.
}

\author{
Walid Soliman Ismail Mahmoud Elsaidy*
}

The purpose of this study was to evaluate and compare the validity and reliability of Harvard step test (HST) to predict the maximum oxygen uptake (Vo2max) through different methods, the first method via traditional Harvard step test (THST) with step height $50.8 \mathrm{~cm}$, the second method by Multi-Height step according to the knee joint angle (KJAHST), where the researcher detects to Criterion-Related Validity between the two methods and the laboratory test via treadmill gas analysis system according to the established protocol ( ZAN 600 device), and in order to achieve the best method to predict (Vo2max) correct values through the Harvard step test in healthy college students, Participants $(N=120)$ age $20.06 \pm 0.56$ years, height $171.79 \pm$ $5.83 \mathrm{~cm}$, weight $70.39 \pm 6.39 \mathrm{~kg}$, the values of vo2max were calculated with the previous methods, the results showed that the value of correlation coefficient between (THST) and laboratory test was $r=0.818$, while the value of correlation coefficient through (KJAHST) and the laboratory test was $r=0.905$, which indicates that the two methods are valid to predict vo2max correct values, but the researcher recommended the usage of (KJAHST) method because it is more valid and accurate than (THST), also there weren't any significant differences between testing and re-testing for the second method (KJAHST) and the value of correlation coefficient was $r=0.913$, so the researcher built Z-scores levels using the second method to be the standard in evaluating (Vo2max).

Keywords: validity, reliability, Harvard step test, maximum oxygen uptake (Vo2max).

\section{Introduction:}

M aximum oxygen uptake $\left(\mathrm{Vo}_{2} \max \right)$ is considered as the best indicator of aerobic fitness. However, because of the strenuous effort required by the participant, measuring $\mathrm{Vo}_{2} \mathrm{max}$ is often neither convenient nor safe for some individuals. Consequently, several field-based sub maximal exercise tests for estimating $\mathrm{Vo}_{2}$ max have been developed in an attempt to provide a simple yet valid method for estimating aerobic fitness where the direct measurement of maximal $\mathrm{Vo}_{2} \mathrm{max}$ is not feasible [4]. The step test is one such test and is considered to be a practical field test for assessing individuals' aerobic fitness.

\footnotetext{
* Lecturer, Physical Education Foundations Department, The Faculty of Physical Education, Abu Qir, Alexandria University, Egypt.
}

The mechanism for step testing assessment of aerobic fitness is based on the varied recovery speed from individual to individual, with well conditioned subjects demonstrating better recuperative powers than untrained subjects (Powers \& Howley 2004) [14]. Post-exercise heart rate (PHR) is the primary parameter for estimating aerobic capacity Step tests vary in stepping cadence, platform height, test duration, number of stages and scoring method [3].The Harvard Step test is a test of aerobic fitness, developed by Brouha et al. (1943) in the Harvard Fatigue Laboratories during WWII [22]. The test can be briefed in a procedure where the athlete steps up and down on the platform at a rate of 30 steps per minute (every two seconds) for 5 minutes or until exhaustion with using the heart rate as a biomarker to define the fitness index for each athlete [22]. The feature of this test is that it is simple to 
conduct and requires minimal equipment. There are many other variations of step tests too that were developed, used and modified by many researchers Brouha et al. (1943) [2], Clarke. (1943) [5], Gallagher and Brouha (1943) [7], Skubic and Hodgkins (1963) [19], Cotton (1971) [6], Chin-Mou Liu and Kuei-Fu Lin (2007) [4]. Who performed many trials to get the HST to its most reliable and constant protocol. As well as the disadvantage of Harvard step test is that biomechanical characteristics vary between individuals. For example, considering that the step height is standard, taller people are at an advantage as it will take less energy to step up onto the step. Body weight has also been shown to be a factor. Testing large groups with this test will be time consuming [3, 12-20]. A lot of those studies proved the impact of the leg length, the step height and the angle of knee joint on vo2max result via Harvard step test for example ,( Ryhming I. (1953)[15], Reedy JD, et.al.(1958)[17], Gideon B. Ariel (1969)[8], Shahnawaz H. (1978)[21]. So, the purpose of this study is to evaluate and compare the validity and reliability of Harvard step test (HST) to predict of the maximum oxygen uptake ( $\left.\mathrm{Vo}_{2} \max \right)$ through different methods, the first method via traditional Harvard step test (THST) with step height $50.8 \mathrm{~cm}$, the second method by Multi-Height step according to the knee joint angle (KJAHST), and compare the results with Laboratory test via treadmill gas analysis system (Zan 600 device).

\section{Methods}

\section{Participants:}

A sample of (120) healthy male college students with age (Mean $=20.06, \mathrm{SD}= \pm 0.56$, Range $=$ 2.03), Height (Mean $=171.29, \mathrm{SD}= \pm 5.83$, Range $=24)$, and Weight $($ Mean $=70.39, \mathrm{SD}=$ \pm 6.39 , Range $=30$ ), they voluntarily participated in this study with informed consent prior to any testing; participants were medically examined for cardiovascular and pulmonary diseases by the faculty medical unit, to ensure that they were physically capable of performing tests.

\section{Step test protocol:}

Participants were asked to complete step test consists of different step heights according to knee joint angle (KAJHST). The student steps up and down on step at a rate of 30 steps per minute for 5 minutes or until exhaustion. Exhaustion is defined as when the student cannot maintain the stepping rate for 15 seconds. The student immediately sits down on completion of the test, and the total numbers of heart beats are counted between 1 to 1.5 minutes after finishing. This is the only measure required if using the short form of the test. If the long form of the test is being conducted, there is an additional heart rate measures at between 2 to 2.5 minutes, and between 3 to 3.5 minutes. The heart rate was calculated with polar stop watch. The Fitness Index score is determined by the following equations. For example, if the total test time was 300 seconds (if completed the whole 5 minutes), and the number of heart beats between 1-1.5 minutes was 90, between 22.5 it was 80 and between 3-3.5 it was 70, then the long form Fitness Index score would be: $(100 \times 300) /(240 \times 2)=62.5$. Note: you are using the total number of heart beats in the 30 second period, not the rate (beats per minute) during that time.

Physical Fitness Index (PFI) (short form) $=(100$ $\mathrm{x}$ test duration in seconds) divided by $(5.5 \mathrm{x}$ pulse count between 1 and 1.5 minutes).

Physical Fitness Index (PFI) (long form) $=(100$ $\mathrm{x}$ test duration in seconds) divided by ( $2 \mathrm{x}$ sum of heart beats in the recovery periods). The PFI obtained in this study was calculated with the equation from the Harvard Step Test (Brouha 1943). [2, 4, 12-20]. in this test The researcher used the same standard of Harvard step test with 
modifying the height of the step according to the knee joint angle as follows: the height of the step should start with $35 \mathrm{~cm}$ and up to be correlated with the knee joint angle when and only when it's $90^{\circ}$ sharp as it will guarantee that all participants are performing the test without any overload according to the height of all participants and to concern the individual differences when the test is performed aiming to raise its validity and reliability.

\section{Maximal exercise test (Laboratory test):}

( $\left.\mathrm{Vo}_{2} \mathrm{max}\right)$ was measured with the treadmill test via (ZAN600 device Treadmill). Bruce protocol (Chin-Mou 2007) was used to measure vo2max [4], which begins with a 3-minutes warm-up during which participants walk comfortably (below $2.8 \mathrm{~km} / \mathrm{hr}, 0 \%$ grade). The workload is then increased by grade and running pace every 3 minutes until participants reach volitional exhaustion. Oxygen uptake was considered maximal if any two of the following criteria were met: (a) volitional fatigue; (b) respiratory exchange ratio $\geq 1.1$; (c) heart rate at or near age-predicted maximum $( \pm 10 \mathrm{bpm}) ;(\mathrm{d}) \leq$ $2 \mathrm{~mL} \cdot \mathrm{kg}-1$ increase in $\mathrm{Vo}_{2}$ with an increase in workload; (e) rating of perceived exertion scale $\geq 18 .[4,10]$.

\section{Procedure:}

Before the two step tests and maximal exercise test were conducted, participants were given a brief on the testing procedures and they had a short practice session to have some experience about the test protocol. After 5 minutes of warm-up, the participants were asked to perform the three tests (1) (THST) with the same step height, (2) (KAJHST) and with different stepping heights a according to the knee joint angle, and (3) maximal exercise test (Laboratory test) in a randomized order. Participants were required to rest for at least 48 hours between any two tests and finished all the tests within 2 weeks. A slow walk recovery and cool-down stretching were provided after all the tests. 


\section{Data analysis:}

The data was collected and statistically treated with SPSS software version 10. The Descriptive statistics (mean, standard deviation and range) of the participants' physical characteristics were computed. A Pearson product moment correlation coefficient was calculated between measured $\left(\mathrm{Vo}_{2} \mathrm{max}\right)$ by (Laboratory test) and
(THST), (KAJHST).and also between test and re-test after one week from the first measurement of (KAJHST) and the paired tsample test was calculated between the test and re-test. And the Bland and Altman [1] approach for limits of agreement were adopted for statistical analysis of the data. And the Z-scores for (KAJHST) were calculated with equation:

[(Grade-mean divided by standard deviation) $x 10]+50$

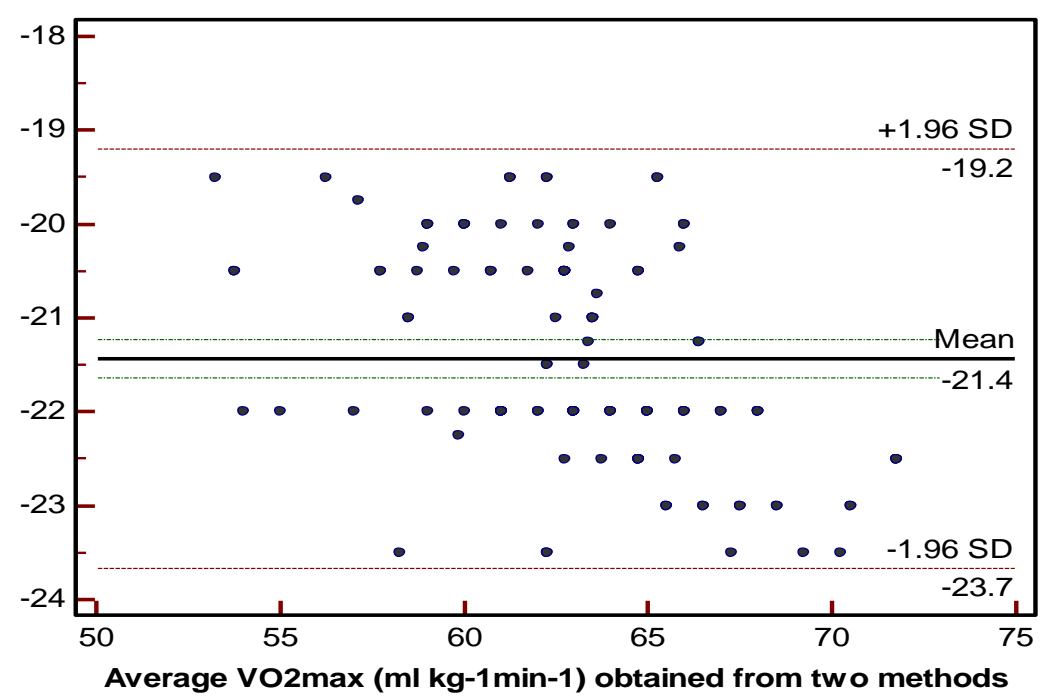

Figure 1: Difference between the VO2max values obtained from direct measurement and those obtained from the (KJAHST) data plotted against their means (Bland and Altman method [1].

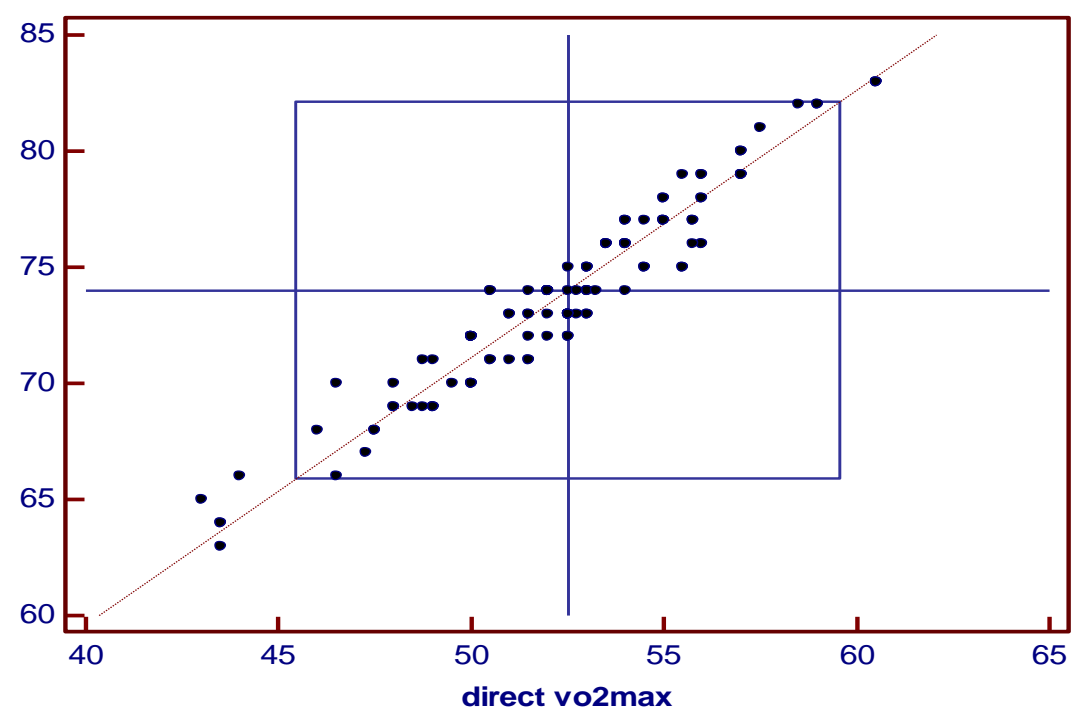

Figure 2: Relationship between the VO2max values obtained from direct measurement and those obtained from the Results: (KJAHST) data 
The above figure $(1,2)$ shows that the values of vo2max in (KJAHST) are correlated to the laboratory test. Analysis of the data using the Bland and Altman (Bland and Altman, 1986 [1]) method for limits of agreement between direct vo2max measurement and predict vo2max via (KJAHST) revealed that the limits of agreement were -19.02 to -23.7 . These limits are small enough that the (KJAHST) can be used confidently in place of the direct method (Figure 1). The limits of agreement analysis suggest that application of the present form of (KJAHST) may be justified for the studied population. A highly significant correlation $(\mathrm{r}=$ 0.929, $\mathrm{P}<0.01)$ existed between the (KJAHST) and VO2max. The vo $\mathrm{vo}_{2} \max$ values and characteristics of participants via Laboratory test, THST and KJAHST were as follows, Laboratory test (Mean $=52.34, \mathrm{SD}= \pm 3.54$, Range $=17.50)$, THST $($ Mean $=68.20, \quad S D=$ \pm 4.90 , Range $=23.00)$, and KJAHST $($ Mean $=$ 73.77, $\mathrm{SD}= \pm 4.07, \quad$ Range $=20.00)$. The correlations between measured $\mathrm{Vo}_{2} \max$ by (Laboratory test) and (THST) and (KAJHST) were $\left(\mathrm{r}=0.818^{* *}\right.$ and $\left.0.905^{* *}\right)$ respectively. Where, Correlation is significant at the 0.01 level. This result showed that the correlation coefficients were higher with (KAJHST) and more valid than (THST). The result showed that there were no significant differences between test and re-test after one week from the first measurement of (KAJHST). And the result had shown that the correlation coefficients between test and re-test were high $(\mathrm{r}=0.929 * *)$ which means that the correlation is significant at (0.01) level between test and re-test for (KJAHST). Also there was no difference between test and re-test for (KJAHST), moreover paired t-sample test value between test and re-test was $(\mathrm{t}=1.47)$ it less $(\mathrm{T}=2.045$ in $\mathrm{DF}=29)$ which proves the reliability of the test. The results showed that the minimum value was (grade 63 facing $34 \mathrm{Z}$ scores) while the maximum value was (grade 83 facing $73 \mathrm{Z}$-scores).

\section{Discussion:}

Better limits of agreement existed between the two methods when the newly developed method was used to predict VO2max from the (KJAHST) data. The limits of agreement when using the new method were -23.67 and -19.20. When using the new method, the (KJAHST) predicted VO2max values for $100 \%$ of the participants fell within the limits of agreement. According to the results the mean and SD values of the VO2max determined by direct method were $52.34 \pm 3.54 \mathrm{ml} / \mathrm{kg}-1 / \mathrm{min}-1$, range $17.50 \mathrm{ml} / \mathrm{kg}-1 / \mathrm{min}-1$. Where minimum value was 43.00 , and maximum value was 60.50 . The mean and SD values of the predicted $\mathrm{VO} 2 \mathrm{max}$ by (THST) were $68.20 \pm 4.90 \mathrm{ml} / \mathrm{kg}-1 / \mathrm{min}-1$, range $23.00 \mathrm{ml} / \mathrm{kg}-1 / \mathrm{min}-1$. Where minimum value was 54.00 , and maximum value was 77.00. The mean and SD values of the predicted VO2max by (KJAHST) was $73.77 \pm 4.07 \mathrm{ml} / \mathrm{kg}$ $1 / \mathrm{min}-1$, range $20.00 \mathrm{ml} / \mathrm{kg}-1 / \mathrm{min}-1$. Where minimum value was 63.00 , and maximum value was 83.00. The results showed that the correlations between the direct and predicted VO2max of all subjects according to the two methods (THST) and (KJAHST) are as follows; $(\mathrm{r}=0.818,0.905)$. So, the Harvard step test is valid to predict of $\mathrm{VO} 2 \mathrm{max}$ which proves the validity of this test according to previous studies (Brouha L, et. al. (1943) [3]. Ryhming I (1953); [17]. Montoye HJ (1953). [12]. Reedy JD, et.al. (1958) [15]. Keen EN, Sloan AW (1958). [9]. Sloan AW. (1959). [20]. Ricci B, et.al. (1966) [16]. Montoye HJ, et.al. (1969) [13]. Meyers CR. (1969) [11]). The repeatability was investigated by having (30) of the subjects perform the test twice. Intra-class correlation coefficients were used to determine the testretest reliability. Where, the correlation coefficients between test -retest were $(\mathrm{r}=$ 0.929). Also, the results showed non-significant differences between the test -retest of the (KJAHST). Where, $\mathrm{t}=-1.47$ ) according to above studies. According to results the Z-scores for 
(KJAHST) were calculated with the following equation:

[(Grade-mean divided by standard deviation) $\mathrm{x}$ 10] +50 . The results showed that the minimum value was (grade 63 facing 34 Z-scores) while the maximum value was (grade 83 facing 73 Zscores).

\section{Conclusion:}

In the present study, the (THST) and (KAJHST) of the step tests correlated well with the measured $\mathrm{Vo}_{2}$ max via Laboratory test, and were found to be valid tests for estimating the aerobic fitness of college students in step tests but it is better to use the (KAJHST) test because it is more valid than the (THST) . Moreover, the step test that used a multi-height step (KAJHST) for college students shown in this study had a coefficient of ( $r=0.905)$, while the step test that used the same height step (THST) for college students shown in this study had a coefficient of $(\mathrm{r}=0.818)$. Additionally, the (KAJHST) for college students showed that the correlation coefficients between test and re-test was $(r=0.929)$. From the results of this study, the researcher suggests that a step test that uses a multi-high step (KAJHST) would be more appropriate for college students.

\section{Recommendations:}

- Using (KAJHST) to predict of the (vo2max) of college students.

- To apply the same study on different age levels for both sexes.

- Building the standard levels of (KAJHST) for different age levels in both sexes.

\section{References:}

1. Bland JM, Altman DG.(1986).Statistical method for assessing agreement between
2. Brouha, Lucien (1943), "The Step Test: A Simple Method for Measuring Physical Fitness for Muscular Work in Young Men", Res.Quart. 14, pp. 31-36.

3. Buono MJ, Roby JJ, Micale FG, Sallis JF, Shepard WE (1991).Validity and reliability of predicting maximum oxygen uptake via field tests in children and adolescents. Pediatr Exerc Sci 3:250-5.

4. Chin-Mou Liu, Kuei-Fu Lin (2007). Estimation of Vo2max a comparative analysis of post- exercise heart rate and physical fitness index from 3-minute step test. J Exerc Sci Fit o Vol 5 o No 2 o 119123.

5. Clarke, H. L. (1943), A Functional Physical Fitness Test for College Women, J. Hlth. Phys. Educ. 14, p. 358 (Abstract).

6. Cotton, Doyice J. (1971), "A Modified Step Test for Group Cardiovascular Testing", Res. Quart. 42, pp. 91-95.

7. Gallagher, J. Roswell, and Brouha, Lucien (1943), "A Simple Method for Testing The Physical Fitness for Boys", Res. Quart. 14, pp. 24-30.

8. Gideon B. Ariel (1969); the effect of knee joint angle on Harvard Step Test Performance. Ergonomics, 12: 33-37.

9. Keen EN, Sloan AW. 1958; Observations on the Harvard step test. J Appl Physiol. 13(2):241-3.

10. Lin JC (1995); The Guide lines of Exercise Physiology Experimentation. Taipei, R.O.C.: Shtabook.

11. Meyers CR., (1969); a study of the reliability of the Harvard step test. Res Q.; 40(2): 423.

12. Montoye HJ. (1953); The Harvard step test and work capacity. Rev Can Biol. 11(5):491-9.

13. Montoye HJ, Willis PW 3rd, Cunningham DA, Keller JB(1969). Heart rate response to a modified Harvard step test: males and females, age 10-69. Res Q.; 40(1):153-62. 
14. Powers SK, Howley ET (2004). Exercise Physiology, 4th edition. New York: McGraw-Hill.

15. Reedy JD, Saiger GL, Hosler RH (1958): Evaluation of the Harvard Step Test with respect to factors of height and weight. Int $\mathrm{Z}$ Angew Physiol.17 (2):115-9.

16. Ricci B, Baldwin K, Hakes R, Fein J, Sadowsky D, Tufts S, Wells C(1966)., Energy cost and efficiency of Harvard steptest performance. Int $\mathrm{Z}$ Angew Physiol.; 22(2):125-30.

17. Ryhming I. (1953). A modified Harvard step test for the evaluation of physical fitness. Arbeitsphysiologies.; 15(3):235-50.
18. Shahnawaz H. (1978); Influence of limb length on a stepping exercise the American Physiological Society.

19. Skubic, Vera and Hodkins, Jean (1963), "Cardiovascular Efficiency for Girls and Women", Res. Quart. 34, pp. 191-198.

20. Sloan AW. (1959); A modified Harvard step test for women. J Appl Physiol. 14:9856.

21. Shahnawaz H. (1978); Influence of limb length on a stepping exercise the American Physiological Society

22. Topendsports: fitness testing: Harvard step test ;http://www.topendsports.com/testing/tests/s tep-harvard.htm. 